\title{
Registro de daño de Aulacoscelis melanocera (Coleoptera: Orsodacnidae) sobre Cycas revoluta (Cycadaceae) en Campeche, México
}

\author{
Record of damage by Aulacoscelis melanocera (Coleoptera: Orsodacnidae) \\ on Cycas revoluta (Cycadaceae) in Campeche, Mexico
}

\author{
SAÚL SÁNCHEZ-SOTO ${ }^{1}$
}

\begin{abstract}
${ }^{1}$ Doctor en Ciencias. Colegio de Postgraduados, Campus Tabasco, Laboratorio de Entomología, Río Seco y Montaña Segunda Sección, Periférico Carlos A. Molina s/n, C. P. 86402, Huimanguillo, Tabasco, México, sssoto@colpos.mx, https://orcid.org/0000-0001-5080-4611.
\end{abstract}

Autor para correspondencia

Saúl Sánchez-Soto. Doctor en Ciencias. Colegio de Postgraduados, Campus Tabasco, Laboratorio de Entomología, Río Seco y Montaña Segunda Sección, Periférico Carlos A. Molina s/n, C. P. 86402, Huimanguillo, Tabasco, México,sssoto@colpos.mx, https://orcid.org/00000001-5080-4611.

\section{Citación sugerida}

SÁNCHEZ-SOTO, S. 2020. Registro de daño de Aulacoscelis melanocera (Coleoptera: Orsodacnidae) sobre Cycas revoluta (Cycadaceae) en Campeche, México. Revista Colombiana de Entomología 46 (2): e7111. https://doi. org/10.25100/socolen.v46i2.7111

Recibido: 10-oct-2018

Aceptado: 17-feb-2020

Publicado: 31-dic-2020

Revista Colombiana de Entomología ISSN (Print): 0120-0488

ISSN (On Line): 2665-4385

https://revistacolombianaentomologia.univalle.edu.co

Open access

(c) (i) (2) (2) BY-NC-SA 4.0

Publishers: Sociedad Colombiana de Entomología SOCOLEN (Bogotá, D. C., Colombia) https://www.socolen.org.co

Universidad del Valle (Cali, Colombia)

https://www.univalle.edu.co

(C) 2020 Sociedad Colombiana de Entomología - SOCOLEN y Universidad del Valle - Univalle
Resumen: El 7 de mayo de 2018, en el jardín de un sitio arqueológico en el norte de Campeche, México, se observó una planta de Cycas revoluta con aproximadamente el $40 \%$ del follaje dañado por un enjambre de Aulacoscelis melanocera (Coleoptera: Orsodacnidae). El presente trabajo constituye el primer registro de esta especie ocasionando daño severo en C. revoluta y su primer registro para el estado de Campeche.

Palabras clave: Cycadales, Cycadaceae, palma sago, Chrysomeloidea, Aulacoscelidinae, daño, Aulacoscelis melanocera, Coleoptera, Orsodacnidae.

Abstract: On May 7, 2018, in the garden of an archaeological site in northern Campeche, Mexico, a Cycas revoluta plant was observed with approximately $40 \%$ of the foliage damaged by a swarm of Aulacoscelis melanocera (Coleoptera: Orsodacnidae). The present work constitutes the first report of this species causing severe damage in C. revoluta and the first record of this insect for the state of Campeche.

Keywords: Cycadales, Cycadaceae, sago palm, Chrysomeloidea, Aulacoscelidinae, damage, Aulacoscelis melanocera, Coleoptera, Orsodacnidae.

\section{Introducción}

El género Aulacoscelis Duponchel y Chevrolat (Coleoptera: Orsodacnidae) comprende 16 especies descritas, distribuidas desde el suroeste de los Estados Unidos hasta Panamá y Colombia (Lawrence y Ślipiński 2014). Aulacoscelis melanocera Duponchel y Chevrolat, 1842 se registra en México, Guatemala, El Salvador, Honduras, Nicaragua y Panamá (Monrós 1954). En México, se encuentra en los estados Yucatán, Oaxaca, Veracruz, Puebla, San Luis Potosí y Tamaulipas (Monrós 1954) y, más recientemente, en el estado de Chiapas (Lázaro-Zermeño et al. 2012). De las siete especies de Aulacoscelis que habitan en México (Monrós 1954; Ordóñez-Reséndiz 2014), A. melanocera es la que presenta distribución más amplia y la única del género registrada en la península de Yucatán, específicamente en el norte del estado de Yucatán (Monrós 1954). Los adultos miden de 7,7 a 8,2 mm, son oblongos, moderadamente convexos y lustrosos; el color general es rojizo, el cual varía de naranja pálido a rojo sangre; las antenas (excepto el segmento basal que es rojizo), tarsos, tibias y ápice de los fémures son de color negro (Monrós 1954). La especie más cercana a $A$. melanocera es $A$. appendiculata Cox y Windsor, 1999 de Panamá, y ambas difieren en la genitalia del macho y en los élitros de las hembras (Cox y Windsor 1999).

Las plantas hospederas del género Aulacoscelis comprenden especies del orden Cycadales (Monrós 1954; Windsor et al. 1999). Las hospederas conocidas para $A$. melanocera son Dioon merolae De Luca, Sabato y Vázquez-Torres, 1981 (Zamiaceae), especie nativa de México (Lázaro-Zermeño et al. 2012), y Cycas revoluta Thunberg, 1783 (Cycadaceae), especie exótica (Monrós 1954). En D. merolae, los adultos se encuentran entre las brácteas de conos femeninos donde horadan la esclerotesta para luego ovipositar en el material de reserva, ya que sus larvas suelen 
alimentarse de este material y del embrión (Lázaro-Zermeño et al. 2012). La información sobre $C$. revoluta, se limita a las etiquetas que acompañan a algunos de los especímenes adultos de A. melanocera examinados por Monrós (1954), procedentes de México (San Luis Potosí) y El Salvador que indican que fueron colectados en esta especie de planta. En el presente trabajo se informa de un daño severo ocasionado por adultos de $A$. melanocera en el follaje de una planta de $C$. revoluta, en una localidad del estado de Campeche, México.

\section{Materiales y métodos}

La planta de $C$. revoluta dañada era parte del jardín del área de recepción de la zona arqueológica de Santa Rosa Xtam-

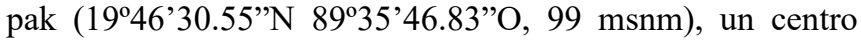
regional Maya remoto y selvático en el norte del estado de Campeche, municipio de Hopelchén, aproximadamente a 120 $\mathrm{km}$ del Golfo de México y $10 \mathrm{~km}$ del límite con el estado de Yucatán (Morales-López y Folan 2005). El clima es cálido subhúmedo con precipitación anual que supera ligeramente los $1.000 \mathrm{~mm}$; la temperatura mínima es de $22,7^{\circ} \mathrm{C}$ (en enero) y la máxima de $29,3^{\circ} \mathrm{C}$ (en mayo); el relieve corresponde a una gran llanura rodeada por lomeríos, algunos de los cuales alcanzan los $150 \mathrm{msnm}$, y la vegetación está compuesta básicamente por selva mediana subcaducifolia con árboles que alcanzan entre 10 y $20 \mathrm{~m}$ de altura (Morales-Valderrama 2004). El jardín ocupaba un área aproximada de 1 ha y estaba compuesto por plantas de guano (Sabal sp.), palma camedor (Chamaedorea elegans Mart., 1830), cedro (Cedrela odorata L., 1759), ceiba (Ceiba pentandra [L.] Gaertn, 1791), almendro (Terminalia catappa L., 1767), cítricos (Citrus spp.) y buganvilia (Bougainvillea sp.), entre otras. También había dos plantas más de $C$. revoluta, de menor tamaño, pero sin daño.

La planta dañada se detectó el 7 de mayo de 2018. El tronco de esta medía aproximadamente $26 \mathrm{~cm}$ de diámetro y $70 \mathrm{~cm}$ de alto, y de acuerdo con la información proporcionada, la planta tenía entre 10 y 15 años. Sobre el follaje se hallaron varios individuos de $A$. melanocera, solos o en grupos, dañando los foliolos. Alrededor del tronco, entre la base de las frondas, había un enjambre compuesto por cientos de individuos que se encontraba oculto entre una capa densa de material compuesto por una cantidad descomunal de filamentos de color marrón claro. Se recolectaron 43 individuos (29 hembras y 14 machos) en frascos con alcohol al $70 \%$ y se identificaron consultando Monrós (1954), y Cox y Windsor (1999). Los especímenes fueron depositados en la colección entomológica del Colegio de Postgraduados, Campus Tabasco. También se obtuvo una muestra de dicho material denso compuesto de filamentos, así como de foliolos dañados y sanos, los cuales fueron desprendidos de la planta mediante un corte en la base con una navaja y observados bajo microscopio estereoscópico.

\section{Resultados y discusión}

El daño abarcó aproximadamente el $40 \%$ de todo el follaje y consistió en el secamiento total de los foliolos de las frondas nuevas ubicadas en el centro de la corona de la planta, y en el secamiento parcial de los foliolos de las frondas adyacentes a esta (Figs. 1A, 1B). Este daño se derivó de las heridas ocasionadas por los adultos de A. melanocera (Fig. 1C).

Al examinar al microscopio estereoscópico varios foliolos dañados, el daño se ubicó en el envés, entre la nervadura central y los bordes curvados de los mismos. La epidermis inferior y el parénquima fueron destruidos, quedando sólo una capa compuesta por la cutícula y la epidermis superior, de modo que el área dañada tuvo la apariencia de un surco longitudinal a ambos lados de la nervadura central. Este daño es semejante al ocasionado por $A$. appendiculata en el follaje de Zamia fairchildiana L. D. Gómez, 1982 en el cual el insecto
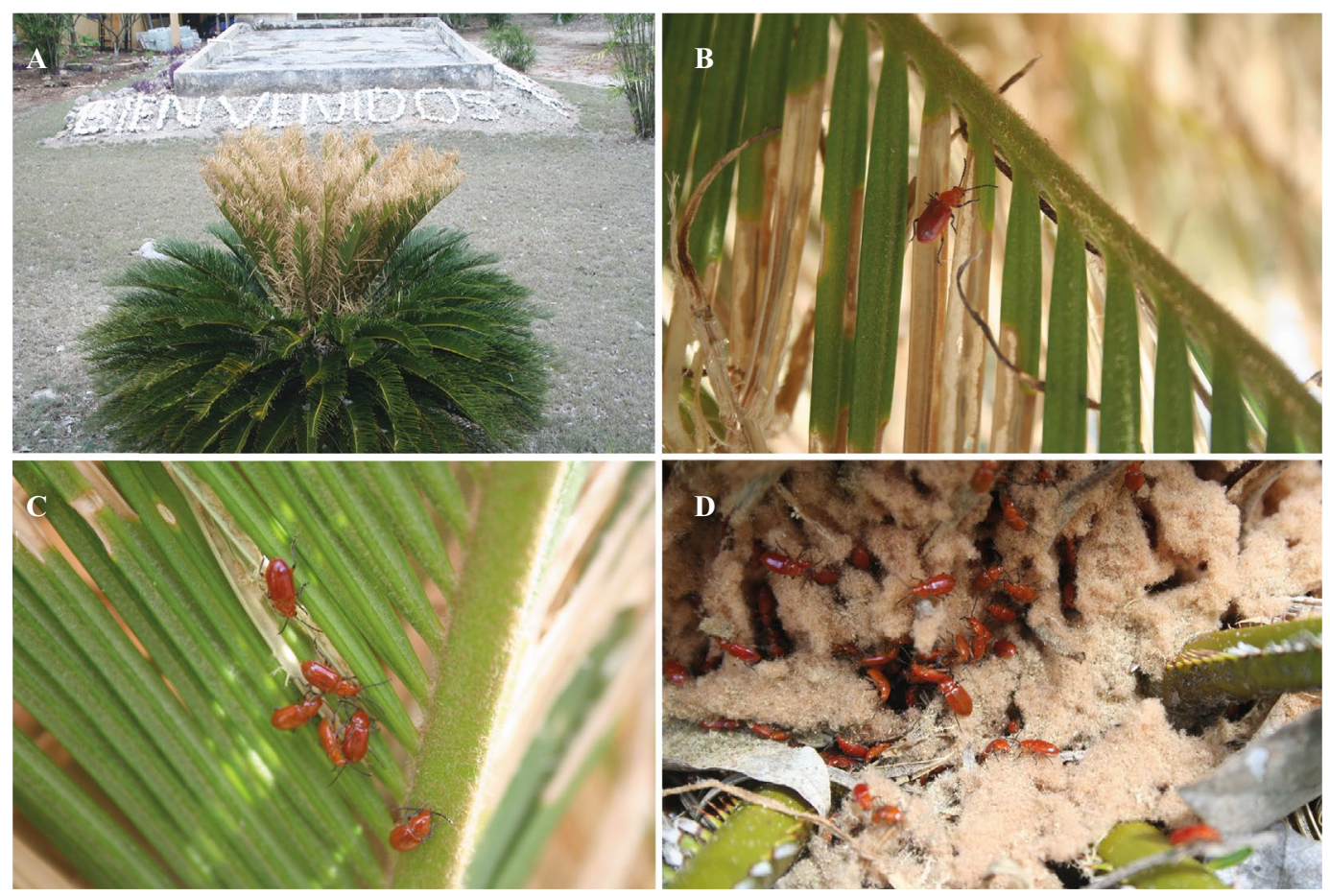

Figura 1. A. Planta de Cycas revoluta dañada por adultos de Aulacoscelis melanocera. B. Fronda con foliolos parcialmente dañados. C. Adultos de A. melanocera dañando foliolos. D. Adultos saliendo de una capa de material denso compuesto de filamentos, ubicada alrededor del tronco, entre la base de las frondas. 
forma un surco para chupar los jugos de la epidermis en lugar de ingerir al por mayor el tejido del parénquima (Windsor et al.1999; Cox y Windsor 1999). La presencia de polen de angiospermas en el intestino de A. melanocera (Crowson 1991) y en las heces de $A$. appendiculata (Prado et al. 2012) sugiere que las especies de Aulacoscelis se alimentan de polen, pero ingieren los fluidos de las cícadas para secuestrar azoxiglucósidos tóxicos que pueden utilizar en su propia defensa ante depredadores (Prado et al. 2011, 2012).

Los filamentos que conformaban la capa de material denso mencionada correspondieron a la pubescencia o tricomas del envés de los foliolos de las frondas dañadas. Es probable que su acumulación en la base de las frondas (Fig. 1D) se haya debido a la propia actividad del enjambre de $A$. melanocera en etapa adulta (Figs. 1D, 2A). Por otro lado, en las muestras de foliolos se observaron varios adultos de $A$. melanocera que acudieron prontamente para ingerir el jugo foliar en el área del corte, tanto en los foliolos desprendidos y colocados sobre una caja de plástico a una distancia aproximada de $2 \mathrm{~m}$ de la planta madre (Fig. 2B), como en esta última (Fig. 2C). Lo anterior indica que por lo menos a corta distancia, los adultos de esta especie tienen la capacidad de detectar con rapidez los componentes del líquido foliar de $C$. revoluta $\tan$ pronto como este fluye o queda expuesto en el exterior del follaje. El daño y comportamiento de $A$. melanocera es similar al de Janbechynea elongata Jacoby, 1888 (Coleoptera: Orsodacnidae) registrado en plantas de C. revoluta cultivadas en el Área Natural Protegida Sierra de Otontepec, Veracruz, ya que este coleóptero también ocasiona el secamiento de las frondas, presenta conducta gregaria, se esconde en el tronco y las frondas, y continúa alimentándose de estas aún después de ser retiradas de la planta madre (Reyes-Ortíz et al. 2016).

No fue posible continuar con las observaciones en los días sucesivos al 7 de mayo, sin embargo, el 15 de julio de 2018 se acudió nuevamente al sitio arqueológico para obser- var la planta dañada. Esta no presentó daños mayores a los observados el 7 de mayo, aunque el follaje dañado estaba mucho más seco. No se observó en ella ningún individuo de $A$. melanocera. Se desconoce si el enjambre fue combatido de alguna manera. Es probable que este haya abandonado la planta poco después del 7 de mayo, y que el período de ataque haya durado pocos días, pues en Costa Rica los enjambres de A. costaricensis Bechyné, 1950 al atacar cícadas lo hacen raras veces por más de tres días (Windsor et al. 1999).

Es factible que el ataque de $A$. melanocera se debió a la presencia de follaje nuevo, pues al parecer existe una sincronización entre la aparición de los enjambres de Aulacoscelis y Janbechynea con la emisión de follaje nuevo de sus hospederas (Windsor et al. 1999; Reyes-Ortíz et al. 2016), lo cual puede estar asociado a la textura suave de los foliolos nuevos, los cuales carecen de la rigidez de las hojas viejas (Windsor et al. 1999; Prado et al. 2014). La ausencia de individuos y de daño de $A$. melanocera en las otras dos plantas de $C$. revoluta del sitio arqueológico posiblemente de debió a que estas no tenían follaje nuevo.

Aunque $C$. revoluta es una planta ornamental cultivada generalmente en jardines de paisajes comerciales y residenciales de áreas urbanas (Marler y Moore 2010; Mayett-Moreno et al. 2014), su presencia en áreas con vegetación boscosa en las cercanías, al parecer la hace vulnerable al ataque de $A$. melanocera y de $J$. elongata (Reyes-Ortíz et al. 2016) en el área de distribución de estas. Se desconoce si los daños ocasionados por A. melanocera pueden conducir a la muerte de plantas de C. revoluta; entre tanto, el daño se puede considerar de tipo estético, y a este insecto como una plaga del paisaje en jardines como el del sitio arqueológico mencionado. Se sugiere realizar estudios que permitan conocer aspectos de la biología y comportamiento de este insecto, lo cual es fundamental para implementar medidas sustentables de control en casos de ataque a esta especie de planta ornamental.
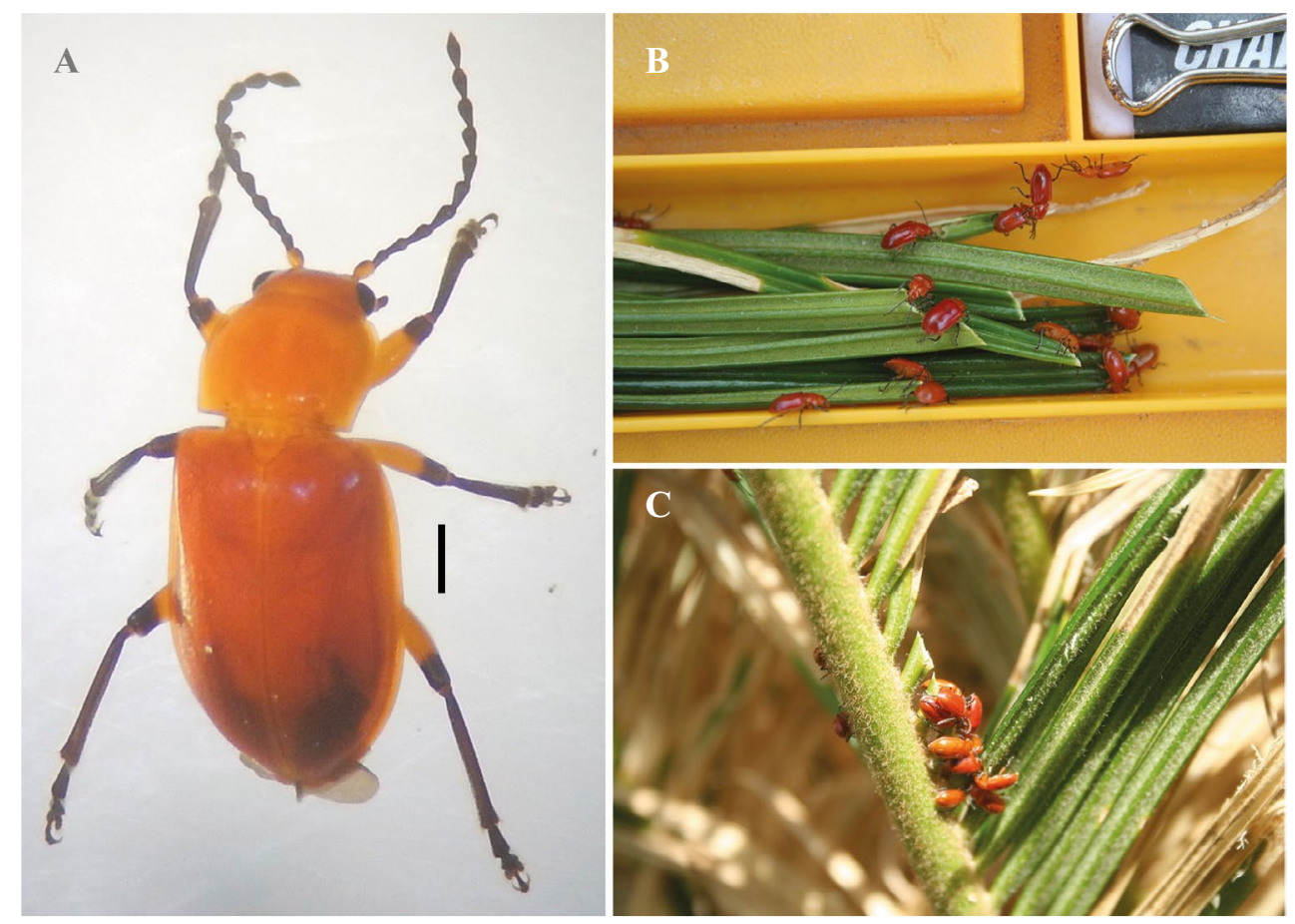

Figura 2. A. Adulto macho de Aulacoscelis melanocera colectado en Campeche (Escala: $1 \mathrm{~mm}$ ). B. Adultos ingiriendo jugo foliar en el área del corte de foliolos desprendidos y, en la base de los mismos en la planta madre de Cycas revoluta $(\mathbf{C})$. 


\section{Agradecimientos}

Al Colegio de Postgraduados, por el apoyo financiero del proyecto 509 del Campus Tabasco: "Fauna asociada a plantas cultivadas en la región sur-sureste de México", del cual se derivó el presente trabajo; y a los evaluadores anónimos por la revisión y sugerencias que mejoraron la presentación del mismo.

\section{Literatura citada}

COX, M. L.; WINDSOR, D. M. 1999. The first instar larva of $A u$ lacoscelis appendiculata $\mathrm{n}$. sp. (Coleoptera: Chrysomelidae: Aulacoscelinae) and its value in the placement of the Aulacoscelinae. Journal of Natural History 33: 1049-1087. https://doi. org/10.1080/002229399300083

CROWSON, R. A. 1991. The relations of Coleoptera to Cycadales. pp. 13-28. En: Zunino, M.; Bellés, X.; Blas, M. (Eds.). Advances in Coleopterology. Asociación Europea de Coleopterología. Barcelona. $323 \mathrm{p}$.

LAWRENCE, J. F.; ŚLIPIŃSKI, A. 2014. Orsodacnidae C. G. Thomson, 1859. pp. 184-189. En: Leschen, R. A. B.; Beutel, R. G. (Eds.). Handbook of Zoology. Arthropoda: Insecta. Coleoptera, Beetles. Volume 3: Morphology and Systematics (Phytophaga). Walter de Gruyter. Berlín, Alemania. 675 p.

LÁZARO-ZERMEÑO, J. M.; GONZÁLEZ-ESPINOSA, M.; MENDOZA, A.; MARTÍNEZ-RAMOS, M. 2012. Natural history of Dioon merolae (Zamiaceae) in Chiapas, México. Botanical Sciences 90 (1): 73-87. https://doi.org/10.17129/botsci.387

MARLER, T. E.; MOORE, A. 2010. Cryptic scale infestations on Cycas revoluta facilitate scale invasions. HortScience 45 (5): 837-839. https://doi.org/10.21273/HORTSCI.45.5.837

MAYETT-MORENO, Y.; SALOMÉ-CASTAÑEDA, E.; BARAJAS-DOMÍNGUEZ, M. I. 2014. Comercialización de cícadas mexicanas (Zamiaceae) en Atlixco, Puebla. Un estudio exploratorio. Revista Mexicana de Ciencias Agrícolas 5 (4): 633-644. https://doi.org/10.29312/remexca.v5i4.924v

MONRÓS, F. 1954. Revision of the chrysomelid subfamily Aulacoscelinae. Bulletin of the Museum of Comparative Zoology at Harvard College 112 (4): 319-360. https://www.biodiversitylibrary. org/page/2830349\#page/344/mode/1up

MORALES-LÓPEZ, A.; FOLAN, W. J. 2005. Santa Rosa Xtampak, Campeche: su patrón de asentamiento del Preclásico al Clásico. Mayab (18): 5-16. https://dialnet.unirioja.es/descarga/articulo/2775113.pdf

MORALES-VALDERRAMA, C. 2004. Identidad y modernización agrícola en Los Chenes, Campeche, México. Perspectivas Latinoamericanas 1: 123-143. https://core.ac.uk/reader/236154870
ORDÓÑEZ-RESÉNDIZ, M. M. 2014. Catálogo de autoridades taxonómicas y base de datos curatorial de la familia Chrysomelidae en México. Universidad Nacional Autónoma de México. Facultad de Estudios Superiores Zaragoza. Informe final, SNIB-CONABIO. PROYECTO No. HS003. México, D. F. 103 p. http://www.conabio.gob.mx/institucion/proyectos/resultados/ InfHS003.pdf

PRADO, A.; LEDEZMA, J.; CUBILLA-RIOS, L.; BEDE, J. C.; WINDSOR, D. M. 2011. Two genera of Aulacoscelinae beetles reflexively bleed azoxyglycosides found in their host cycads. Journal of Chemical Ecology 37: 736-740. https://doi. org/10.1007/s10886-011-9977-5

PRADO, A.; MCKENNA, D. D.; WINDSOR, D. 2012. Molecular evidence of cycad seed predation by immature Aulacoscelidinae (Coleoptera: Orsodacnidae). Systematic Entomology 37 (4): 747-757. https://doi.org/10.1111/j.1365-3113.2012.00639.x

PRADO, A.; SIERRA, A.; WINDSOR, D.; BEDE, J. C. 2014. Leaf traits and herbivory levels in a tropical gymnosperm, Zamia stevensonii (Zamiaceae). American Journal of Botany 101 (3): 437447. https://doi.org/10.3732/ajb.1300337

REYES-ORTÍZ, J. L.; LUNA-FERRER, J. M.; GONZÁLEZ-GÁNDARA， C.; CRUZ-MORALES， G. E.; DOMÍNGUEZ-BARRADAS, C. 2016. Herbivoría en cícadas (Cycadophyta) por adultos de Janbechynea elongata Jacoby, 1888 (Coleoptera: Orsodacnidae) en el ANP Sierra de Otontepec, Veracruz. Acta Zoológica Mexicana (nueva serie) 32 (1): 126-128. https://doi.org/10.21829/azm.2016.3201938

WINDSOR, D.; NESS, J.; DIEGO-GÓMEZ, L.; JOLIVET, P. H. 1999. Species of Aulacoscelis Duponchel and Chevrolat (Chrysomelidae) and Nomotus Gorham (Languriidae) feed on fronds of Central American cycads. The Coleopterists Bulletin 53 (3): 217-231.

\section{Origen y financiación}

El presente trabajo derivó del proyecto de investigación "Fauna asociada a plantas cultivadas en la región sur-sureste de México", el cual se encuentra vigente, es financiado por el Colegio de Postgraduados y se encuentra registrado en la matriz de investigación del Campus Tabasco con la clave 509.

\section{Contribución de los autores}

El único autor del trabajo, Saúl Sánchez-Soto, realizó el trabajo de campo y de identificación de la especie de insecto en laboratorio, asi como la elaboración del manuscrito. 